\title{
NOUVELLE
}

\section{Le cancer de la prostate conduit à la découverte d'un nouveau rétrovirus infectieux humain}

Jean-Luc Darlix, Marc Sitbon

\author{
J.L. Darlix : Laborétro, Unité de virologie humaine, IFR128, \\ École Normale Supérieure de Lyon et Inserm \\ 46, allée d'Italie, 69364 Lyon, France. \\ Jean-Luc.Darlix@ens-lyon.fr \\ M. Sitbon : Institut de Génétique Moléculaire de Montpellier \\ (IGMM), CNRS, IFR 122, \\ 1919, route de Mende, 34293 Montpellier Cedex 5, France. \\ sitbon@igmm.cnrs.fr
}

\section{$>$ Oncovirus et cancers}

Depuis le milieu du XXe siècle, de nombreuses études expérimentales sur des virus humains et animaux liés au cancer, alors appelés «oncovirus», ont ouvert la voie à des découvertes majeures dans le domaine des mécanismes moléculaires du vivant, avec en particulier la découverte des premiers gènes impliqués dans les processus d'oncogenèse. II s'agit là des fameux proto-oncogènes et de leurs variants oncogènes qui faisaient une entrée très remarquée dans le champ fondamental, pour devenir ensuite des marqueurs diagnostiques, et des cibles thérapeutiques [1, 2].

À l'heure actuelle, un faisceau d'arguments indique qu'environ $25 \%$ des cancers ont une origine virale [3], avec comme cause directe des composants de certaines souches de papillomavirus humains comme les HPV 16 et 18 [4]. Quand elle est indirecte, la cancérogenèse viro-induite implique des modifications du fonctionnement de protéines clefs de la prolifération et de la survie cellulaires, voire des altérations chromosomiques des cellules infectées. Ainsi, la modification des voies de mort cellulaire par apoptose semble jouer un rôle majeur dans le développement de carcinomes hépatocellulaires dus au virus de l'hépatite C (VHC), en particulier par la protéine virale Core [5]. Par ailleurs, de nombreux réarrangements chromosomiques ont été décrits dans les leucémies induites par le rétrovirus leucémogène humain HTLV-1 (le premier rétrovirus humain découvert), et un rôle direct de la protéine virale
Tax dans ces altérations est suspecté [6]. Des modifications génétiques liées à l'insertion de tout ou partie d'un génome rétroviral (mutagenèse insertionnelle) ont été décrites dans de nombreux modèles animaux comme étant responsables de processus cancéreux, alors que chez l'homme, de telles insertions n'ont pas été retrouvées dans des infections naturelles, mais lors d'essais thérapeutiques impliquant un vecteur rétroviral dérivé d'un rétrovirus MLV (murine leukemia virus) [7].

\section{Rétrovirus et cancers}

Les Rétrovirus forment une large classe de virus enveloppés à ARN qui ont été, depuis le début du XXe siècle, reconnus comme pouvant être la cause de cancers et de déficits immunitaires. Initialement découverts chez les oiseaux et les rongeurs, des rétrovirus infectieux ont été retrouvés chez un grand nombre de vertébrés. Les récentes découvertes des HTLV puis des VIH, notamment celle du VIH-l, l'agent causal du Sida chez l'homme, ont donné la mesure du caractère ubiquitaire de ces infections virales. Parmi les rétrovirus de mammifères, des oncorétrovirus de la famille des MLV, regroupés sous le terme de gammarétrovirus ( $\gamma$-rétrovirus), ont été isolés chez des souris, chats, singes, porcs, koalas, etc. De très nombreuses études expérimentales sur les MLV ont permis de découvrir les enzymes clés de la réplication et de la dissémination rétrovirales que sont la protéase (PR), la reverse transcriptase (RT) et l'intégrase (IN), ainsi que la première protéine virale chaperon d'ARN - la NC - (pour revues, voir $[8,9])$. Ces études ont aussi montré que les MLV peuvent modifier l'expression des proto-oncogènes, directement au moyen d'un rapt - connu sous le terme de «transduction génétique »-, et de les transformer en oncogènes vectorisés par un MLV recombinant appelé DLV (defective leukemia virus). De très nombreux oncogènes ont ainsi été identifiés et clonés à partir des DLV qui les portent. L'oncogenèse causée par un MLV peut être aussi indirecte en intervenant par mutagenèse insertionnelle comme indiqué ci-dessus, et causant la trans-activation d'un protooncogène cellulaire après intégration de I'ADN proviral, issu de la transcription inverse du génome par la RT [10].

\section{Cancer de la prostate et $\boldsymbol{\gamma}$-rétrovirus}

Avec environ 40000 nouveaux cas diagnostiqués chaque année en France, le cancer de la prostate constitue le cancer ayant la plus haute incidence chez I'homme de plus de 50 ans. Jusqu'à une date très récente, on pensait que les $\gamma$ rétrovirus de type MLV n'infectaient pas naturellement l'homme. Or, deux articles récents par Urisman et al. [11], et Dong et al. [12], montrent de façon remarquable qu'un virus apparenté aux MLV, appelé XMRV (Xenotropic murine leukemia related virus), est retrouvé dans les cellules stromales associées aux cellules tumorales de patients atteints d'un cancer de la prostate [11-13]. Les auteurs établissent un lien entre ce XMRV et une prédisposition familiale pour ce type de cancer, connue sous le nom de locus 
$\mathrm{HPCl}$, affectant le gène codant la RNAseL. Tout porte à croire qu'il s'agit, dans le cas de cette pathologie, d'une mutation unique, la R4620, fréquente dans la population humaine, avec des valeurs d'environ $13 \%$ à l'état homozygote, et de 35-60\% à l'état hétérozygote [14]. La mutation R4620 semble diminuer d'un facteur environ 3 l'activité RnaseL [14], qui est un effecteur majeur de la réponse interféron, elle-même clef de voûte des réponses innées anti-virales [15]. Les auteurs rapportent que la plus faible activité de la RNaseL mutée est nécessaire pour que l'infection par le XMRV devienne chronique dans des cellules humaines ex vivo [12]. Pour autant, les relations causales entre la présence de XMRV et ce type de cancer ne sont pas établies pour l'instant.

Néanmoins, XMRV, ce nouveau rétrovirus humain, possède toutes les caractéristiques des MLV, un génome unique avec les gènes codant Gag (protéines de structure du virion), Pol (les enzymes PR, RT et IN) et Env (les protéines d'enveloppe de surface et transmembranaires). Aux extrémités de l'ARN génomique, on retrouve les séquences non-codantes 5'et 3' UTR (untranslated region) contenant tous les signaux nécessaires à la réplication et à l'expression transcriptionnelle et traductionnelle du génome dans les cellules. Toutefois l'enveloppe est de type xénotrope et reconnaît un récepteur présent à la surface des cellules humaines, XPRI, dont la forme allèlique chez les rongeurs ne permet pas leur infection [16]. Le récepteur XPRI a de nombreux domaines transmembranaires, comme nombre de récepteurs de rétrovirus, et serait couplé à la transduction du signal via les protéines $G$, et donc à l'activation du métabolisme cellulaire au début de l'infection. Les premières études moléculaires concernant les sites d'intégration chromosomique du XMRV ont été menées à bien dans deux cas de cancer de la prostate homozygotes pour R462Q. Les données confirment que le XMRV s'est bien répliqué dans le tissu épithélial en plus des cellules stromales, et que l'ADN viral s'est intégré au voisinage de gènes codant d'importants facteurs de transcription, à l'image de ce qui a déjà été largement décrit avec les MLV [12].

Ces observations remarquables mettent en exergue une fois de plus que les rétrovirus circulent au sein de - et entrepopulations, d'autant plus qu'ils peuvent se répliquer à un haut niveau comme le VIH-1. La présence du XMRV soulève de nombreuses interrogations, à commencer par son origine, sa variabilité, son mode de transmission, son incidence dans la population générale, mais aussi les déterminants moléculaires du virus [17] et de l'hôte responsables de pathologies comme le cancer de la prostate.

Ces questions primordiales auront certainement l'heur d'occuper virologues, généticiens, cancérologues, cliniciens, thérapeutes, et épidémiologistes et, sans doute, environnementalistes et diététiciens. $\diamond$ Prostate cancer leads to the discovery of a new human infectious retrovirus

\section{RÉFÉRENCES}

1. Huebner RJ, Todaro GJ. Oncogenes of RNA tumor viruses as determinants of cancer. Proc Natl Acad Sci USA 1969; 64 : 1087-94.
2. Hanahan D, Weinberg RA. The hallmarks of cancer. Cell $2000 ; 100: 57-70$.

3. Pagano JS, Blaser M, Buendia MA, et al. Infectious agents and cancer: criteria for a causal relation. Semin Cancer Biol $2004 ; 14:$ 453-71.

4. Munger K, Howley PM. Human papillomavirus immortalization and transformation functions. Virus Res $2002 ; 89$ : 213-28.

5. Moradpour D, Penin F, Rice CM. Replication of hepatitis C virus. Nat Rev Microbiol 2007 ; 5 : 453-63.

6. Marriott SJ, Semmes 0J. Impact of HTLV-I Tax on cell cycle progression and the cellular DNA damage repair response. Oncogene 2005 ; 24 : 5986-95.

7. Thrasher AJ, Hacein-Bey-Abina S, Gaspar HB, et al. Failure of SCID-XI gene therapy in older patients. Blood 2005 ; 105 : 4255-7.

8. Darlix JL, Cristofari G, Rau M, et al. Nucleocapsid protein of human immunodeficiency virus as a model protein with chaperoning functions and as a target for antiviral drugs. Adv Pharmacol $2000 ; 48: 345-72$

9. Sitbon M, Denesvre C, Dardalhon V, et al. Les rétrovirus leucémogènes murins : pathogènes, gènes et outils génétiques. Virologie $2001 ; 5: 265-93$.

10. Rosenberg N, Jolicoeur P. Retroviruses. In : Coffin J, Hughes SH, Varmus HE, eds. Cold Spring Harbor, NY: Cold Spring Harbor Laboratory Press, 1997 : 475-585.

11. Urisman A, Molinaro RJ, Fischer N, et al. Identification of a novel gammaretrovirus in prostate tumors of patients homozygous for R462Q RNASEL variant. PLoS Pathog 2006 ; 2 : e25.

12. Dong B, Kim S, Hong $S$, et al. An infectious retrovirus susceptible to an IFN antiviral pathway from human prostate tumors. Proc Natl Acad Sci USA 2007 ; $104: 1655-60$.

13. Fan H. A new human retrovirus associated with prostate cancer. Proc Natl Acad Sci USA 2007 ; $104: 1449-50$.

14. Casey G, Neville PJ, Plummer SJ, et al. RNASEL Arg462GIn variant is implicated in up to $13 \%$ of prostate cancer cases. Nat Genet $2002 ; 32$ : 581-3.

15. Thakur CS, Jha BK, Dong B, et al. Small-molecule activators of RNase $L$ with broad-spectrum antiviral activity. Proc Natl Acad Sci USA 2007 ; 104 : 9585-90.

16. Battini JL, Rasko JE, Miller AD. A human cell-surface receptor for xenotropic and polytropic murine leukemia viruses: possible role in $\mathrm{G}$ protein-coupled signal transduction. Proc Natl Acad Sci USA 1999; $96: 1385-90$

17. Corbin A, Prats AC, Darlix JL, Sitbon M. A nonstructural gag-encoded glycoprotein precursor is necessary for efficient spreading and pathogenesis of murine leukemia viruses. J Virol $1994 ; 68: 3857-67$.

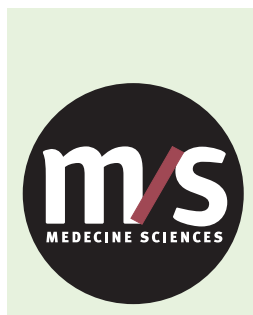

Tarifs d'abonnement M/S - 2007

Abonnez-vous

à Médecine/Sciences
> Depuis 20 ans, grâce à $m / s$, vous vivez en direct les progrès des sciences biologiques et médicales

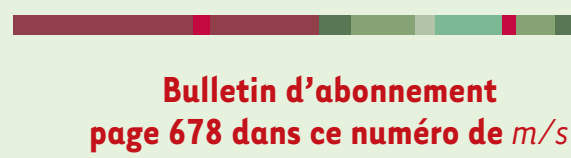

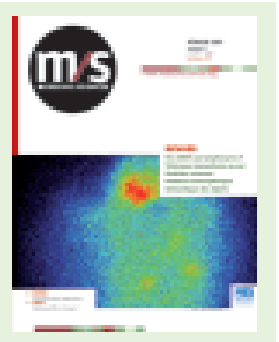

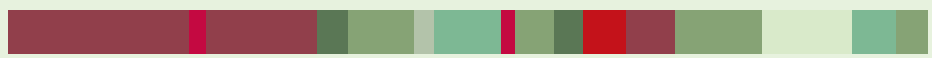

